\title{
ON STATIONARY VACUUM SOLUTIONS TO THE EINSTEIN EQUATIONS
}

\author{
MICHAEL T. ANDERSON
}

\section{IntRoduction.}

A stationary space-time $(M, g)$ is a 4-manifold $M$ with a smooth Lorentzian metric $g$, of signature $(-,+,+,+)$, which has a smooth 1 -parameter group $G \approx \mathbb{R}$ of isometries whose orbits are time-like curves in $M$. We assume throughout the paper that $M$ is a chronological space-time, i.e. $M$ admits no closed time-like curves, c.f. $\S 1.1$ for further discussion.

Let $S$ be the orbit space of the action $G$. Then $S$ is a smooth 3-manifold and the projection

$$
\pi: M \rightarrow S
$$

is a principle $\mathbb{R}$-bundle, with fiber $G$. The chronology condition implies that $S$ is Hausdorff and paracompact, c.f. [Ha] for example. The infinitesimal generator of $G \approx \mathbb{R}$ is a time-like Killing vector field $X$ on $M$, so that

$$
\mathcal{L}_{X} g=0
$$

The metric $g=g_{M}$ restricted to the horizontal subspaces of $T M$, i.e. the orthogonal complement of $\langle X\rangle \subset T M$ then induces a Riemannian metric $g_{S}$ on $S$. Since $X$ is non-vanishing on $M$, $X$ may be viewed as a time-like coordinate vector field, i.e. $X=\partial / \partial t$, where $t$ is a global time function on $M$. The time function $t$ gives a global trivialization of the bundle $\pi$ and so induces a diffeomorphism from $M$ to $\mathbb{R} \times S$. The metric $g_{M}$ on $M$ may be then written globally in the form

$$
g_{M}=-u^{2}(d t+\theta)^{2}+\pi^{*} g_{S}
$$

where $\theta$ is a connection 1 -form for the $\mathbb{R}$-bundle $\pi$ and

$$
u^{2}=-\langle X, X\rangle>0 \text {. }
$$

The 1 -form $\xi$ dual to $X$ is thus given by $\xi=-u^{2}(d t+\theta)$. The 1 -form $\theta$ is uniquely determined by $g_{M}$ and the time function $t$, but of course changes by an exact 1-form if the trivialization of $\pi$ is changed. We point out that $\left(M, g_{M}\right)$ is geodesically complete as a Lorentzian manifold if and only if $\left(S, g_{S}\right)$ is complete as a Riemannian manifold, c.f. Lemma 1.1.

The vacuum Einstein field equations on the space-time $(M, g)$ are

$$
r_{M}=0,
$$

where $r_{M}$ is the Ricci curvature of $\left(M, g_{M}\right)$.

Stationary vacuum space-times are usually considered as the possible final, i.e. time-independent, states of evolution of a physical system, in particular isolated physical systems such as isolated stars or black holes, outside regions of matter. The most important non-trivial example is the Kerr metric, c.f. $[\mathrm{W}]$, modeling the time-independent gravitational field outside a rotating star.

It is easy to see from the field equations, c.f.(1.4) below, that there are no non-flat stationary vacuum solutions of the field equations (0.3) whose orbit space is a closed 3-manifold $S$. Hence, we will always assume that $S$ is an open 3 -manifold.

Partially supported by NSF Grant DMS 9802722. 
Next, it is natural to consider the class of stationary vacuum space-times which are geodesically complete. In this respect, Lichnerowicz [L, $\S 90]$ proved that any such solution $(M, g)$ for which the 3-manifold $\left(S, g_{S}\right)$ is complete and asymptotically flat is necessarily flat Minkowski space.

The assumption that $S$ is asymptotically flat is very common in general relativity in that such space-times serve as natural models for isolated physical systems, e.g. stars or black holes. The reasoning here is that as one moves further and further away from an isolated gravitational source, the corresponding gravitational field should decay as it does in Newtonian gravity, giving in the limit of infinite distance the empty Minkowski space-time.

However, mathematically the requirement that $S$ is asymptotically flat is a very strong assumption on both the topology and geometry of $S$ outside large compact sets. Further, the reasoning above is not at all rigorous. It presupposes that a geodesically complete stationary solution of the vacuum equations, i.e. a stationary solution without sources, is necessarily empty, and so in particular flat.

Consider the fact that there are geodesically complete, non-stationary vacuum space-times consisting of gravitational waves, c.f. [MTW, §35.9] or [R, §8.8] for example. Again, physically, such space-times can be considered as idealized limiting configurations at infinite distance from radiating sources. Similarly, if there does in fact exist a complete non-flat stationary vacuum solution, say $\left(M_{\infty}, g_{\infty}\right)$, then there could well exist models $(M, g)$ for isolated physical systems which are asymptotic to $\left(M_{\infty}, g_{\infty}\right)$ at space-like infinity. For instance, it is not even clear apriori that the curvature of a stationary space-time, vacuum outside a compact source region, should decay anywhere at infinity.

The first main result of this paper is that in fact there are no such non-trivial stationary spacetimes; this of course places the physical reasoning above on stronger footing.

Theorem 0.1. Let $(M, g)$ be a geodesically complete, chronological, stationary vacuum space-time. Then $(M, g)$ is the flat (i.e. empty) Minkowski space $\left(\mathbb{R}^{4}, \eta\right)$, or a quotient of Minkowski space by a discrete group $\Gamma$ of isometries of $\mathbb{R}^{3}$, commuting with $G$. In particular, $M$ is diffeomorphic to $S \times \mathbb{R}, d \theta=0$ and $u=$ const.

This result, together with Lemma 1.1 below implies that if $(M, g)$ is a non-flat stationary vacuum space-time, then the orbit space $S$ must have a non-empty metric boundary. More precisely, since $\left(S, g_{S}\right)$ is Riemannian, let $\bar{S}$ denote the metric, (equivalently the Cauchy), completion of $S$ and let $\partial S=\bar{S} \backslash S$. Hence

$$
\Sigma=\partial S \neq \emptyset
$$

if $(M, g)$ is not flat.

In order to avoid trivial ambiguities, we will only consider maximal stationary quotients $S$. For example any domain $\Omega$ in $\mathbb{R}^{3}$ with the flat metric, $u$ a positive constant, and $\theta=0$ generates a stationary vacuum solution, (namely a domain in Minkowski space). In this case, the metric boundary $\partial \Omega$ is artificial, and has no intrinsic relation with the geometry of the solution. The solution obviously extends to a larger domain, i.e. all of Minkowski space. Thus, we only consider maximal solutions $\left(S, g_{S}, u, \theta\right)$, in the sense that the data $\left(S, g_{S}, u, \theta\right)$ does not extend to a larger domain $\left(S^{\prime}, g_{S}^{\prime}, u^{\prime}, \theta^{\prime}\right) \supset\left(S, g_{S}, u, \theta\right)$ with $u^{\prime}>0$ on $M^{\prime}$. It follows that in any neighborhood of a point $q \in \Sigma=\partial S$, either the metric $g_{S}$ or the connection 1-form $\theta$ degenerates in some way, or $u$ approaches 0 in some way, or both.

Without any further restrictions, the behavior of the data near $\partial S$ can be quite complicated; numerous concrete examples of this can be found among the axi-symmetric stationary, or even axisymmetric static, i.e. Weyl, solutions; c.f. [A1] for further discussion. In particular, singularities, both of curvature type and of non-curvature type, may form at the boundary. The horizon $H=$ $\{u=0\}$, viewed as a subset of $S$, may or may not be well-defined in this generality; of course it 
corresponds to the locus in $M$ where the Killing vector $X$ becomes null. Even when $H$ is well-defined and smooth, in general there may be other, possibly singular, parts to $\partial S$.

Theorem 0.1 leads to the following apriori estimate on the norm of the curvature of a stationary vacuum solution away from the boundary of $S$, and on the rate of curvature blow-up on approach to the boundary.

Theorem 0.2. There is a constant $K<\infty$ such that if $(M, g)$ is any chronological stationary vacuum solution, (not geodesically complete), then

$$
\left|R_{M}\right|[x] \leq K / \rho^{2}[x],
$$

where $R_{M}$ is the curvature tensor of $(M, g),[x]$ is the Killing orbit through $x \in M$ and $\rho(x)=$ dist $_{g_{S}}([x], \partial S)$. The constant $K$ is independent of the data $(M, g)$.

Note that Theorem 0.2 implies Theorem 0.1 by letting $\rho \rightarrow \infty$. On the other hand, Theorem 0.2 requires Theorem 0.1 for its proof. In particular, this result shows that if $\partial S$ is compact in the completion $\bar{S}$, then the curvature of $(M, g)$ decays at least quadratically w.r.t. the distance from $\partial S$.

The contents of the paper are as follows. We discuss some background information and preliminary results in $\S 1$, needed for the work to follow. Theorem 0.1 is proved in $\S 2$ and Theorem 0.2 is proved in $\S 3$.

I would like to thank Piotr Chrusciel and Jim Isenberg for useful discussions, the referee for pointing out some needed clarifications and Grisha Perelman for pointing out an error in a previous version of the paper.

\section{Background and Preliminary Results.}

§1.1. A stationary space-time $(M, g)$ uniquely determines the orbit data $\left(S, g_{S}, u, \Omega\right)$ described in $\S 0$, where $\Omega=d \theta$ is the curvature 2-form of the bundle $\pi$ on $S$. Conversely, given arbitrary orbit data $\left(S, g_{S}, u, \Omega\right), u>0$, satisfying certain equations, (c.f. (1.3)-(1.6) below), there is a unique stationary space-time $(M, g)$ in the sense of $\S 0$, i.e. a chronological space-time with a global isometric $\mathbb{R}$-action with the given orbit data.

Of course, if $(M, g)$ is not chronological, then it will not be uniquely determined by the orbit data. One may for instance take a $\mathbb{Z}$-quotient of $(M, g)$, preserving the orbit data. More importantly, if $(M, g)$ is not chronological, then the orbit space $S$ may not be a manifold; even if $S$ is a manifold, it may not be Hausdorff, c.f. [Ha]. Since the arguments to follow are global on $S$, we require that $S$ is globally well-behaved, which is ensured by the chronology condition. It is not known for instance if Theorem 0.1 is valid without this assumption.

Recall that a space-time $(M, g)$ is geodesically complete if all geodesics in $(M, g)$, parametrized by an affine parameter $s$, are defined for all $s \in \mathbb{R}$. The vertical subspace of $T M$ is the subspace spanned by the Killing field $X$ and the horizontal distribution $\mathcal{H}$ is its orthogonal complement in $T M$, defined by the metric $g_{M}$.

Lemma 1.1. A stationary space-time $\left(M, g_{M}\right)$ is geodesically complete if and only if the orbit space $\left(S, g_{S}\right)$ is geodesically complete.

Proof: Suppose $\left(M, g_{M}\right)$ is geodesically complete. Let $\gamma$ be a geodesic in $S$. Since the projection $\pi: M \rightarrow S$ is a principle fiber bundle, with horizontal spaces $\mathcal{H} \subset T M$, the geodesic $\gamma$ may be lifted to a horizontal geodesic $\bar{\gamma}$ in $\left(M, g_{M}\right)$, with the same parametrization. Since $\left(M, g_{M}\right)$ is complete, $\bar{\gamma}$ is defined for all values of the parameter, and hence so is $\gamma$.

Conversely, suppose $\left(S, g_{S}\right)$ is geodesically complete, and hence complete as a metric space. Let $\gamma$ be a geodesic in $M$, with affine parameter $s$ and tangent vector $T$. Then the projection $\sigma=\pi \circ \gamma$ 
is a curve in $S$, whose acceleration is given by

$$
\nabla_{V} V=\frac{1}{2} \kappa^{2} \nabla u^{-2}-\frac{1}{2} \kappa L(V) .
$$

Here $V=d \sigma / d s=\pi_{*} T, \nabla$ is the covariant derivative in $\left(S, g_{S}\right), \kappa=<X, T>=$ const and $L$ is the linear map defined by $\left\langle L(A), B>X=[\mathrm{A}, B]^{v}\right.$ where $A, B$ are horizontal vector fields on $M$ and $v$ is the vertical projection, c.f. [T, Ch.18.3] for example. Conversely, any curve $\sigma$ satisfying (1.1) lifts to a geodesic in $(M, g)$.

The equations (1.1) form a $2^{\text {nd }}$ order system of ODE w.r.t. the parameter $s$; note that $L(V)$ is linear in $V$, while $\kappa$ is a constant in $s$, depending linearly on $V$. By local existence and uniqueness, there exist locally defined solutions $\sigma$ for arbitrary initial data $(x, V(x)) \in T S$. Since $S$ is complete, it follows that $\sigma$ exists for all values of $s \in \mathbb{R}$. Hence $(M, g)$ is geodesically complete.

Remark 1.2. It is easy to verify that if $(M, g)$ is a stationary, (strongly) globally hyperbolic space-time, in the sense that $(M, g)$ admits a geodesically complete Cauchy surface $L$, (w.r.t. the induced metric), then $(M, g)$ is geodesically complete. The converse issue however, i.e. whether a chronological, stationary and geodesically complete space-time is necessarily globally hyperbolic, is not clear to the author, at least without further assumptions on $u$ and $\theta$.

For brevity, we will often say that $\left(M, g_{M}\right)$ or $\left(S, g_{S}\right)$ is complete instead of geodesically complete.

$\S 1.2$. Let $\xi=-u^{2}(d t+\theta)$ be the 1 -form dual to the Killing vector $X$, as in $\S 0$. The twist potential $\omega$ is the 1 -form on $M$ defined by

$$
\omega=\frac{1}{2} *(\xi \wedge d \xi)
$$

It is easily verified that $\omega$ is $G$-invariant, and that it descends to a 1 -form $\omega$ on the base space $S$. The form $\omega$ represents the obstruction to integrability of the horizontal distribution in $T M$, and so is related to the curvature 2 -form $\Omega$ of the connection 1 -form $\theta$. In fact, one easily verifies that

$$
2 \omega=-u^{4} * d \theta=-u^{4} * \Omega,
$$

on $\left(S, g_{S}\right)$.

The vacuum Einstein equations (0.3) on $(M, g)$ are $G$-invariant, and so also descend to equations on $S$. The vacuum equations are equivalent to the following equations on $\left(S, g_{S}\right)$ :

$$
\begin{gathered}
r=\frac{1}{u} D^{2} u+2 u^{-4}\left(\omega \otimes \omega-|\omega|^{2} \cdot g\right), \\
\Delta u=-2 u^{-3}|\omega|^{2} \\
\operatorname{div} \omega=3\langle\operatorname{dlog} u, \omega\rangle \\
d \omega=0
\end{gathered}
$$

Here $r=r_{S}$ is the the Ricci curvature of $\left(S, g_{S}\right), D^{2} u$ is Hessian of $u$ on $\left(S, g_{S}\right), \Delta u=t r_{g_{S}} D^{2} u$ and $\log$ is the natural logarithm; we refer for instance to $[\mathrm{Kr}, \mathrm{Ch} .16]$ for a derivation of these equations, (but note that $[\mathrm{Kr}]$ does not use the factor $\frac{1}{2}$ in (1.2)). The equation (1.3) comes from the pure space-like (or horizontal) part of $r_{M}$, the equation (1.4) from the vertical part of $r_{M}$, i.e. $r_{M}(X, X)$, while the equations (1.5)-(1.6) come from the mixed directions. The equation (1.6) implies that $\omega$ is locally exact, i.e. there exists $\phi$, the twist potential, such that

$$
2 \omega=d \phi
$$

locally. On the universal cover $\widetilde{S}$ of $S,(1.7)$ holds globally. 
Observe that these equations are invariant under the substitutions

$$
u \rightarrow \lambda u, \omega \rightarrow \lambda^{2} \omega,
$$

corresponding to $\xi \rightarrow \lambda \xi$, and $\theta \rightarrow \lambda^{-2} \theta$.

$\S 1.3$. To prove Theorems 0.1 and 0.2 , we will need to study sequences of stationary (vacuum) solutions, where all the data $\left(S, g_{S}, u, \omega\right)$ are allowed to vary. Thus, in effect, we need to understand aspects of the moduli space of stationary solutions. For this, we will frequently use the following two Lemmas, which will be proved together.

Lemma 1.3. (Convergence). Let $\left(\Omega_{i}, g_{i}, u_{i}, \omega_{i}\right)$ represent data for a sequence of solutions to the stationary vacuum equations (0.1). Suppose on the domains $\left(\Omega_{i}, g_{i}\right)$,

$$
\left|r_{i}\right| \leq \Lambda, \quad \operatorname{diam} \Omega_{i} \leq D, \text { vol } \Omega_{i} \geq \nu_{o}
$$

and

$$
\operatorname{dist}\left(x_{i}, \partial \Omega_{i}\right) \geq \delta
$$

for some $x_{i} \in \Omega_{i}$ and positive constants $\nu_{o}, \Lambda, D, \delta$. Then, for any $\varepsilon=\varepsilon(\delta)>0$ sufficiently small, there are domains $U_{i} \subset \Omega_{i}$, with $\varepsilon / 2 \leq \operatorname{dist}\left(\partial U_{i}, \partial \Omega_{i}\right) \leq \varepsilon$, and $x_{i} \in U_{i}$ such that a subsequence of the Riemannian manifolds $\left(U_{i}, g_{i}, x_{i}\right)$ converges, in the $C^{\infty}$ topology, modulo diffeomorphisms, to a limit manifold $(U, g, x)$, with limit base point $x=\lim x_{i}$.

Further, the potentials $u_{i}$ and 1-forms $\omega_{i}$ may be renormalized by scalars $\lambda_{i}$, as in (1.8), so that they converge smoothly to limit potential $u$ and 1-form $\omega$. The limit $(U, g, x, u, \omega)$ represents a smooth solution to the stationary vacuum equations.

Lemma 1.4. (Collapse). Let $\left(\Omega_{i}, g_{i}, u_{i}, \omega_{i}\right)$ represent data for a sequence of solutions to the stationary vacuum equations (0.1). Suppose on the domains $\left(\Omega_{i}, g_{i}\right)$,

$$
\left|r_{i}\right| \leq \Lambda, \quad \operatorname{diam} \Omega_{i} \leq D, \text { vol } \Omega_{i} \rightarrow 0
$$

and

$$
\operatorname{dist}\left(x_{i}, \partial \Omega_{i}\right) \geq \delta
$$

for some $x_{i} \in \Omega_{i}$ and constants $\Lambda, D, \delta$. Then, for any $\varepsilon=\varepsilon(\delta)>0$ sufficiently small, there are domains $U_{i} \subset \Omega_{i}$, with $\varepsilon / 2 \leq \operatorname{dist}\left(\partial U_{i}, \partial \Omega_{i}\right) \leq \varepsilon$ with $x_{i} \in U_{i}$, such that $U_{i}$ is either a Seifert fibered space or a torus bundle over an interval. In both cases, the $g_{i}$-diameter of any fiber $F$, (necessarily a circle $S^{1}$ or torus $\left.T^{2}\right)$, goes to 0 as $i \rightarrow \infty$, and $\pi_{1}(F)$ injects in $\pi_{1}\left(U_{i}\right)$.

Consequently, the universal cover $\widetilde{U}_{i}$ of $U_{i}$ does not collapse and hence has a subsequence converging smoothly to a limit $(\widetilde{U}, g, x)$, with $x=\lim x_{i}^{\prime}, x_{i}^{\prime}$ a lift of $x_{i}$ to $\widetilde{U}_{i}$. In addition, the limit $(\widetilde{U}, g, x)$ admits a free isometric $\mathbb{R}$-action.

As above, the potentials $u_{i}$ and 1-forms $\omega_{i}$, after possible renormalization by scalars, converge smoothly to limits $u$ and $\omega$. The limit $(\widetilde{U}, g, x, u, \omega)$ is a smooth solution of the stationary vacuum equations, and all data are invariant under a free isometric $\mathbb{R}$-action on $\widetilde{U}$.

Proofs: The proofs of the first parts of Lemmas 1.3 and 1.4 are essentially immediate consequences of the well-known Cheeger-Gromov theory on convergence and collapse of Riemannian manifolds with bounded curvature, c.f. [CG1,2], [Ka], $[\mathrm{A} 3, \S 2]$ for example. We note that we are implicitly using the fact, special to dimension 3, that the full curvature is determined by the Ricci curvature.

More precisely, under the bounds (1.9)-(1.10), one obtains convergence of a subsequence of $\left\{g_{i}\right\}$ to a $C^{1, \alpha}$ limit metric $g$ on the domain $U$; the convergence is in the $C^{1, \alpha^{\prime}}$ topology, for any $\alpha^{\prime}<\alpha<$ 1. For a clear introduction to this theory, c.f. [P, Ch. 10]. In particular, the bounds (1.9) imply 
a lower bound on the injectivity radius of every point in $U_{i}$; this is Cheeger's lemma, c.f. [C], [P, 10.4.5]

Under the bounds (1.11)-(1.12), the sequence of domains collapses with bounded curvature in the sense that the injectivity radius at every point in $U_{i}$ tends to 0 . This implies that the domains $U_{i}$ admit an F-structure, [CG1,2]. In dimension 3, this means that $U_{i}$ is topologically a graph manifold, i.e. a union of Seifert fibered spaces ( $S^{1}$ fibrations over a surface) or torus bundles over an interval, glued together along toral boundary components of such, c.f. [Ro, §3]. A result of Fukaya, c.f. [F, Ch.11,12] and references therein, implies that on domains of bounded diameter, i.e. under (1.11)-(1.12), for $i$ sufficiently large, the F-structure may be chosen to be pure, so that $U_{i}$ itself is either a Seifert fibered space or a torus bundle over an interval. The collapse takes place by shrinking the fibers, (circles or tori), to points. From the theory of Seifert fibered spaces, c.f. [O] or [Ro, Thm. 4.3], the fibers inject in $\pi_{1}$ whenever $U_{i}$ is not covered by $S^{3}$. But this is necessarily the case here, since $U_{i}$ is an open domain, (c.f. the remark following (0.3). Thus, one may unwrap the collapse by passing to covers, for instance the universal cover, that unwind the fibers. This ability to unwrap collapse on domains of controlled diameter is special to dimension 3.

It remains to show that the convergence is actually smooth $\left(C^{\infty}\right)$, and that the limit, in either case of Lemma 1.3 or 1.4 , is a smooth solution to the stationary vacuum equations. This is done by showing that the equations (1.3)-(1.6) form essentially an elliptic system and using elliptic regularity.

By taking the trace of (1.3) and using (1.4), one derives that

$$
s=-6 u^{-4} \cdot|\omega|^{2},
$$

where $s$ is the scalar curvature of $\left(S, g_{S}\right)$, so that (1.4) is equivalent to

$$
\Delta u=\frac{s}{3} u \text {. }
$$

Since, by hypothesis, the Ricci curvature is uniformly bounded on $\left(\Omega_{i}, g_{i}\right)$, so is the scalar curvature $s_{i}$. Now the potential functions $u_{i}$ may be unbounded, or converge to 0 , in neighborhoods of the base points $x_{i}$. Thus, we renormalize $u_{i}$ by setting

$$
\bar{u}_{i}=u_{i} / u\left(x_{i}\right),
$$

so that $\bar{u}_{i}\left(x_{i}\right)=1$. The equation (1.14) is of course invariant under this renormalization. Moreover, since $u_{i}>0$ everywhere, and since the local geometry of $\left(\Omega_{i}, g_{i}\right)$ is uniformly controlled in $C^{1, \alpha}$ away from $\partial \Omega_{i}$, i.e. within $U_{i}$, the Harnack inequality, (c.f. [GT, Thm. 8.20]), applied to the elliptic equation (1.14) implies that there is a constant $\kappa>0$, independent of $i$, such that

$$
\kappa \leq \frac{s u p \bar{u}_{i}}{\text { inf }_{i}} \leq \kappa^{-1}
$$

here the sup and inf are taken over $U_{i}$, or more precisely over an $\varepsilon / 4$ thickening of $U_{i}$. Of course the diameter bound in (1.9) or (1.11) is being used here. It then follows from $L^{2}$ elliptic theory, c.f. [GT, Thm. 9.11], that the functions $\bar{u}_{i}$ are uniformly bounded in $L^{2, p}\left(U_{i}\right), p<\infty$. Next, as in (1.15), we renormalize the twist 1 -forms $\omega_{i}$ by

$$
\bar{\omega}_{i}=\omega_{i} /\left(u\left(x_{i}\right)\right)^{2}
$$

c.f. (1.8). It then follows from (1.13), (1.15), (1.17) and the uniform $L^{\infty}$ bound on $s_{i}$ that the forms $\bar{\omega}_{i}$ are uniformly bounded in $L^{\infty}$ on $U_{i}$.

Next, to obtain higher regularity, consider the equations (1.5)-(1.6)

$$
\Delta \phi_{i}=3\left\langle d \log \bar{u}_{i}, d \phi_{i}\right\rangle,
$$

locally, i.e. in neighborhoods where the twist potential $\phi=\phi_{i}$ is defined; (we omit the overbar from the notation for $\phi$ ). We may add a constant to $\phi_{i}$ and assume $\phi_{i}\left(x_{i}\right)=0$. By the bound on $\bar{\omega}_{i}$ above, $\left|d \phi_{i}\right|$ is uniformly bounded, as is $\left|d \log \bar{u}_{i}\right|$, so by elliptic regularity, $\phi_{i}$ is bounded locally 
in $L^{2, p}$, and hence $\bar{\omega}_{i}$ is uniformly bounded locally in $L^{1, p}$ everywhere in $U_{i}$. By (1.13) again, this implies $s_{i}$ is bounded in $L^{1, p}$, and so by elliptic regularity applied to (1.14), $\bar{u}_{i}$ is uniformly bounded locally in $L^{3, p}$. Hence, the right side of (1.3) is bounded in $L^{1, p}$, and so the Ricci curvature $r_{i}$ is uniformly controlled locally in $L^{1, p}$ everywhere in $U_{i}$. This implies that the metrics $g_{i}$ are uniformly controlled in $L^{3, p}$ in local harmonic coordinates, c.f. [A3. §3] for example. Hence, by the Sobolev embedding theorem, the sequence $\left\{g_{i}\right\}$ is uniformly bounded in $C^{2, \alpha}, \alpha<1$.

This process may now be iterated inductively to give uniform $C^{k}$ control on $\left\{g_{i}\right\}$, for any $k<\infty$, away from the boundary, as well as uniform $C^{k}$ control on $\left\{\bar{u}_{i}\right\}$ and on $\left\{\bar{\omega}_{i}\right\}$. This proves that the convergence to the limit is in the $C^{\infty}$ topology, as well as $C^{\infty}$ convergence to limits $\bar{u}$ and $\bar{\omega}$. Since the metrics $g_{i}$ are stationary vacuum solutions, it is obvious that the limit $(U, g, \bar{u}, \bar{\omega})$ is also.

As an application of these results, we prove the following Lemma, which shows that a given complete stationary vacuum solution gives rise to another one with uniformly bounded curvature.

Lemma 1.5. Let $(S, g, u, \omega), g=g_{S}$, represent data for a complete non-flat stationary vacuum solution. Then there exists another complete non-flat stationary vacuum solution given by data $\left(S^{\prime}, g^{\prime}, u^{\prime}, \omega^{\prime}\right), g^{\prime}=g_{S^{\prime}}^{\prime}$, obtained as a geometric limit at infinity of $(S, g)$, which has uniformly bounded curvature, i.e.

$$
\left|r_{g^{\prime}}\right| \leq 1 \text { and }\left|r_{g^{\prime}}\right|(y)>0
$$

for some $y \in S^{\prime}$.

Proof: We may assume that $(S, g)$ itself has unbounded curvature, for otherwise there is nothing to prove since (1.18) can then be obtained by a fixed rescaling of $(S, g)$ if necessary. Let $\left\{x_{i}\right\}$ be a sequence in $S$ such that

$$
|r|\left(x_{i}\right) \rightarrow \infty, \text { as } i \rightarrow \infty
$$

Let $B_{i}=B_{x_{i}}(1)$ and let $d_{i}(x)=\operatorname{dist}\left(x_{i}, \partial B_{i}\right)$. Consider the scale-invariant ratio $\left(d_{i}^{2} \cdot|r|\right)(x)$, for $x \in B_{i}$, and choose points $y_{i} \in B_{i}$ realizing the maximum value of $\left(d_{i}^{2} \cdot|r|\right)(x)$ on $B_{i}$. Since $\left(d_{i}^{2} \cdot|r|\right)(x)$ is 0 on $\partial B_{i}, y_{i}$ is in the interior of $B_{i}$. By (1.19), we have

$$
d_{i}^{2}\left(y_{i}\right) \cdot|r|\left(y_{i}\right) \rightarrow \infty, \text { as } i \rightarrow \infty
$$

and so in particular $|r|\left(y_{i}\right) \rightarrow \infty$.

Now consider the pointed rescaled sequence $\left(B_{i}, g_{i}, y_{i}\right)$, where

$$
g_{i}=|r|\left(y_{i}\right) \cdot g \text {. }
$$

By construction, $\left|r_{i}\right|\left(y_{i}\right)=1$, where $r_{i}$ is the Ricci curvature of $g_{i}$. This, together with (1.20) and its scale-invariance, implies that $\delta_{i}\left(y_{i}\right) \equiv \operatorname{dist}_{g_{i}}\left(y_{i}, \partial B_{i}\right) \rightarrow \infty$. Further, by the maximality property of $y_{i}$

$$
\left|r_{i}\right|(x) \leq\left|r_{i}\right|\left(y_{i}\right) \cdot \frac{\delta_{i}(x)}{\delta_{i}\left(y_{i}\right)}=\frac{\delta_{i}(x)}{\delta_{i}\left(y_{i}\right)}
$$

It follows from (1.20) that $\left|r_{i}\right|(x) \leq 2$, at all points $x$ of uniformly bounded $g_{i}$-distance to $y_{i}$, (for $i$ sufficiently large, depending on $\left.\operatorname{dist}_{g_{i}}\left(x, y_{i}\right)\right)$.

If the pointed sequence $\left(B_{i}, g_{i}, y_{i}\right)$, (or a subsequence), is not collapsing at $y_{i}$, i.e. the volume of the unit $g_{i}$-ball at $y_{i}$ is bounded below as $i \rightarrow \infty$, then by Lemma $1.3,\left\{\left(B_{i}, g_{i}, y_{i}\right)\right\}$ has a subsequence converging, smoothly and uniformly on compact subsets, to a limit $\left(U^{\prime}, g^{\prime}, y\right), y=\lim$ $y_{i}$. The limit is a complete stationary vacuum solution, (since $\delta_{i}\left(y_{i}\right) \rightarrow \infty$ ), and by the smooth convergence, $\left|r_{g^{\prime}}\right| \leq 2$ everywhere and $\left|r_{g^{\prime}}(y)\right|=1$, where $y=\lim y_{i}$. A further bounded rescaling then gives (1.18). The limit potential $u$ and twist form $\omega$ are obtained as in Lemma 1.3. 
On the other hand, suppose this sequence is collapsing at $y_{i}$, so that the volume of the unit $g_{i}$-ball at $y_{i}$ converges to 0 , (in some subsequence). Then by Lemmas 1.3 and 1.4 , it is collapsing everywhere within $g_{i}$-bounded distance to $y_{i}$, i.e. within $\left(B_{y_{i}}(R), g_{i}\right)$, for any fixed $R<\infty$. For any such $R$, if $i$ is sufficiently large, there are domains $U_{i}(R) \subset B_{y_{i}}(R)$, with $\partial U_{i}(R)$ near $\partial B_{y_{i}}(R)$ w.r.t. $g_{i}$, which are highly collapsed along an injective Seifert fibered structure or torus bundle structure on $U_{i}(R)$. Hence the universal cover $\left(\widetilde{U}_{i}(R), \widetilde{g}_{i}\right)$ is not collapsing. For any sequence $R_{j} \rightarrow \infty$, there is then a suitable diagonal subsequence $U_{i_{j}}$ such that the covers $\widetilde{U}_{i_{j}}$ converge smoothly, as above, to a complete stationary vacuum solution; again a bounded rescaling then gives (1.18).

\section{Proof of Theorem 0.1.}

Let $\left(M, g_{M}\right)$ be a complete stationary vacuum solution. As above in $\S 1.2$ and $\S 1.3$, we will work exclusively on the 3-manifold quotient $S$, with data $u, \omega$ and $g$ satisfying the field equations (1.3)(1.6). By passing to the universal cover, we may and will assume for this section that $S$ is simply connected.

It is very useful to rewrite the metric $g_{M}$ in (0.1) in the form

$$
g_{M}=-u^{2}(d t+\theta)^{2}+\frac{1}{u^{2}} \bar{g}_{S}
$$

where $\bar{g}_{S}$ is the conformally equivalent metric

$$
\bar{g}_{S}=u^{2} \cdot g_{S}
$$

on $S$. Using standard formulas for behavior under conformal changes, c.f. [B, Ch. 1J], w.r.t this metric the field equations (1.3)-(1.5) are equivalent to:

$$
\begin{gathered}
\bar{r}=2(\operatorname{dlog} u)^{2}+2 u^{-4}(\omega)^{2}, \\
\bar{\Delta} \log u=-2 u^{-4}|\omega|^{2}, \\
\operatorname{div} \omega=4\langle d \log u, \omega\rangle,
\end{gathered}
$$

c.f. also $[\mathrm{Kr}, \mathrm{Ch} .16]$. All metric quantities in (2.3)-(2.5) are w.r.t. the $\bar{g}=\bar{g}_{S}$ metric.

There are two reasons for preferring $\bar{g}$ to $g=g_{S}$. First, it is apparent from (2.3) that

$$
\bar{r} \geq 0
$$

so that $(S, \bar{g})$ has non-negative Ricci curvature. Second, the field equations (2.3)-(2.5) are exactly the Euler-Lagrange equations for the functional

$$
S_{e f f}=\int_{S}\left(s-\frac{1}{2}\left(\frac{\left|d u^{2}\right|^{2}+|d \phi|^{2}}{u^{4}}\right)\right) d V .
$$

Here we are using the fact that $S$ is simply connected, so that the relation (1.7) holds globally on $S$. This functional is the Einstein-Hilbert functional on $G$-invariant metrics on $M$, dimensionally reduced to a functional on data $(\bar{g}, u, \phi)$ on $S$, when $g_{M}$ is expressed in the form (2.1). It corresponds to a coupling of 3-dimensional gravity to the energy (or $\sigma$-model) of the mapping $E=\left(\phi, u^{2}\right)$ from $S$ to the hyperbolic plane. The mapping $E$ is called the Ernst potential and the Euler-Lagrange equations (2.3)-(2.5) imply that

$$
E:\left(S, \bar{g}_{S}\right) \rightarrow\left(H^{2}(-1), g_{-1}\right)
$$

is a harmonic map. Here $H^{2}(-1)$ is the hyperbolic plane, given as the upper half-plane $\left(\mathbb{R}^{2}\right)^{+}=$ $\{(x, y): y>0\}$, with metric

$$
g_{-1}=\frac{d x^{2}+d y^{2}}{y^{2}}
$$


We refer for instance to [H1] or [H2] for further details and discussion on $S_{\text {eff }}$.

From the equation (2.3), we see that

$$
\bar{r}=\frac{1}{2} E^{*}\left(g_{-1}\right)
$$

In particular, the energy density of $e(E)$ of $E$, given by

$$
e(E)=\frac{1}{2}\left|E_{*}\right|^{2}
$$

satisfies

$$
\bar{s}=e(E)=\frac{1}{2} \operatorname{tr}_{\bar{g}} E^{*}\left(g_{-1}\right) .
$$

For clarity, we break the proof up at this stage into two steps.

Step I. Assume the metric $\left(S, \bar{g}_{S}\right)$ is complete.

The space $\left(S, \bar{g}_{S}\right)$ may or may not have uniformly bounded curvature, i.e. possibly after a bounded rescaling,

$$
|\bar{r}| \leq 1
$$

everywhere on $S$, where the norm is taken w.r.t. $\bar{g}_{S}$. If $(2.12)$ holds, then the arguments below are applied to $\left(S, \bar{g}_{S}\right)$. If instead the curvature of $\left(S, \bar{g}_{S}\right)$ is unbounded, (and hence $\left(S, \bar{g}_{S}\right)$ is not flat), we apply Lemma 1.5 to obtain a new non-flat stationary space-time $\left(S^{\prime}, \bar{g}_{S^{\prime}}, u^{\prime}, \omega^{\prime}\right)$ satisfying (2.12). The arguments below are then applied to $\left(S^{\prime}, \bar{g}_{S^{\prime}}\right)$.

With this understood, we drop the prime from the notation and assume that $\left(S, \bar{g}_{S}\right)$ satisfies (2.12).

We now apply the well-known Bochner formula, c.f. [EL, (3.12)], to the harmonic Ernst map E, to obtain

$$
\bar{\Delta} e(E)=|\bar{\nabla} D E|^{2}+\left\langle r_{M}, E^{*}\left(g_{-1}\right)\right\rangle-\sum_{i, j=1}^{3}\left(E^{*} R_{-1}\right)\left(e_{i}, e_{j}, e_{j}, e_{i}\right) .
$$

Here the sign of the curvature tensor for the last term is such that $R_{-1}(X, Y, Y, X)$ is the sectional curvature of $g_{-1}$ for an orthonormal pair $(X, Y)$. We claim that the last two terms in (2.13) are given by

$$
\begin{gathered}
\left\langle\bar{r}, E^{*}\left(g_{-1}\right)\right\rangle=2|\bar{r}|^{2}, \\
-\left(E^{*} R_{g_{-1}}\right)\left(e_{i}, e_{j}, e_{j}, e_{i}\right)=4\left(\bar{s}^{2}-|\bar{r}|^{2}\right) \geq 0 .
\end{gathered}
$$

The equation (2.14) follows immediately from (2.10). For (2.15), using the fact that $g_{-1}$ is of constant sectional curvature -1 , we have $-\left(E^{*} R_{-1}\right)\left(e_{i}, e_{j}, e_{j}, e_{i}\right)=g_{-1}\left(E_{*} e_{i}, E_{*} e_{i}\right) \cdot g_{-1}\left(E_{*} e_{j}, E_{*} e_{j}\right)-$ $g_{-1}\left(E_{*} e_{i}, E_{*} e_{j}\right)^{2}$. Choosing $\left\{e_{i}\right\}$ to be an orthonormal basis in $\left(S, \bar{g}_{S}\right)$ diagonalizing the Ricci curvature $\bar{r}$, and using (2.10), gives (2.15).

In particular, the equations (2.13)-(2.15) show that the energy density $e(E)$ is a subharmonic function on $\left(S, \bar{g}_{S}\right)$.

Since (2.12) holds on $\left(S, \bar{g}_{S}\right),(2.11)$ implies that $e(E)$ is uniformly bounded above on $\left(S, \bar{g}_{S}\right)$. Thus, let $\left\{x_{i}\right\}$ be a maximizing sequence for $e(F)$, i.e.

$$
e(F)\left(x_{i}\right) \rightarrow \sup e(F)<\infty .
$$

Since the curvature of $\left(S, \bar{g}_{S}\right)$ is bounded, and this space is complete, it follows from elementary properties of the Laplacian that

$$
\Delta e(F)\left(x_{i}\right) \leq \varepsilon_{i},
$$


where $\varepsilon_{i} \rightarrow 0$, as $i \rightarrow \infty$. However, (2.13)-(2.15) then imply that

$$
|\bar{r}|^{2}\left(x_{i}\right) \leq \varepsilon_{i} \rightarrow 0 \text {. }
$$

This of course forces $e(E)\left(x_{i}\right)=\bar{s}\left(x_{i}\right) \rightarrow 0$. Since $x_{i}$ is a maximizing sequence, this is only possible if

$$
e(E) \equiv 0
$$

i.e. $E$ is a constant map. This means that $u=$ const $>0, \phi=$ const, and hence $(M, g)$ is flat. Thus $(M, g)$ is Minkowski space, (since $S$ is simply connected).

Observe that this argument now implies that the passage to the geometric limit $\left(S^{\prime}, \bar{g}_{S^{\prime}}\right)$ at the beginning of Step I was not in fact necessary.

Step II. We now remove the assumption that $\bar{g}$ is complete, by transfering the estimates above back to the complete manifold $\left(S, g_{S}\right)$.

Exactly as in the beginning of Step I however, since $\left(S, g_{S}\right)$ is complete, if necessary we use Lemma 1.5 first to pass to a non-flat geometric limit $\left(S^{\prime}, g_{S^{\prime}}\right)$ with uniformly bounded $g^{\prime}$-curvature, i.e. satisfying (1.18). As before, we drop the prime from the notation below.

Since $\bar{g}_{S}=u^{2} g_{S}$, we have the following relation between the Laplacians of $g_{S}$ and $\bar{g}_{S}$, c.f. [B, Ch. 1J] for example:

$$
\bar{\Delta} f=u^{-2} \Delta f+u^{-3}\langle d u, d f\rangle,
$$

for any function $f$, where metric quantities on the right are w.r.t. $g_{S}$. Setting $f=\bar{s}$ then gives

$$
\Delta \bar{s}=u^{2} \bar{\Delta} \bar{s}-\langle d \log u, d \bar{s}\rangle .
$$

Now the function $\bar{s}$ may well be an unbounded function on $\left(S, g_{S}\right)$; (in fact the unboundedness may cause the incompleteness of $\left.\bar{g}_{S}\right)$. However, in terms of the metric $g$, we have

$$
\bar{s}=u^{-2}\left(2|d \log u|^{2}+\frac{1}{2} u^{-4}|d \phi|^{2}\right) \equiv u^{-2} \cdot h,
$$

where the last inequality defines $h$ and the norms on the right are w.r.t. $g_{S}$. This follows by taking the trace of $(2.3)$.

Since the curvature of $g_{S}$ is uniformly bounded, i.e. (1.18) holds, the same arguments as in the proof of Lemma 1.3-1.4 imply that

$$
|d \log u|^{2}+u^{-4}|d \phi|^{2} \leq C
$$

for some $C<\infty$. The estimate (2.19) can also be deduced directly from (1.13) and (1.3)-(1.7). Hence, $h$ is uniformly bounded above on $\left(S, g_{S}\right)$.

Returning to (2.17), we then have

$$
\Delta \bar{s}=\Delta u^{-2} h=u^{-2} \Delta h+h \Delta u^{-2}+2\left\langle d u^{-2}, d h\right\rangle .
$$

Now

$$
\Delta u^{-2}=-2 u^{-3} \Delta u+6 u^{-4}|d u|^{2}=u^{-6}|d \phi|^{2}+6 u^{-4}|d u|^{2},
$$

where the last equality uses (1.4) and (1.7). Hence, combining (2.20)-(2.21), we obtain

$$
\Delta h=u^{2} \Delta \bar{s}-\left(u^{-4}|d \phi|^{2}+6 u^{-2}|d u|^{2}\right) h-2 u^{2}\left\langle d u^{-2}, d h\right\rangle .
$$

Substituting (2.17) gives

$$
\Delta h=u^{4} \bar{\Delta} \bar{s}-\left(u^{-4}|d \phi|^{2}+6 u^{-2}|d u|^{2}\right) h-2 u^{2}\left\langle d u^{-2}, d h\right\rangle-u^{2}\langle d \log u, d \bar{s}\rangle .
$$

Since $\bar{s}=u^{-2} \cdot h, d \bar{s}=-2 u^{-3} h d u+u^{-2} d h$, and so (2.22) becomes

$$
\Delta h=u^{4} \bar{\Delta} \bar{s}-\left(u^{-4}|d \phi|^{2}+6 u^{-2}|d u|^{2}\right) h+4\langle d \log u, d h\rangle+2 u^{-2} h|d u|^{2}-\langle d \log u, d h\rangle,
$$

i.e.

$$
\Delta h=u^{4} \bar{\Delta} \bar{s}-\left(u^{-4}|d \phi|^{2}+4 u^{-2}|d u|^{2}\right) h+3\langle d \log u, d h\rangle .
$$


By (2.18) again, the middle term on the right above equals $-2 h^{2}=-2 u^{4} \bar{s}^{2}$. Hence, we have

$$
\Delta h-3\langle d \log u, d h\rangle=u^{4} \bar{\Delta} \bar{s}-2 u^{4} \bar{s}^{2} .
$$

On the other hand, from the Bochner formula (2.13) and (2.14)-(2.15), we have

$$
\bar{\Delta} \bar{s}=|\bar{\nabla} D E|^{2}+2|\bar{r}|^{2}+4\left(\bar{s}^{2}-|\bar{r}|^{2}\right),
$$

where all quantities are w.r.t. the $\bar{g}$ metric. Substituting this in (2.23) then gives

$$
\Delta h-3\langle d \log u, d h\rangle=u^{4}|\bar{\nabla} D E|^{2}+2 u^{4}\left(\bar{s}^{2}-|\bar{r}|^{2}\right) \geq 0,
$$

where the terms on the left are in the $g$ metric while those on the right are in the $\bar{g}$ metric.

We now basically repeat the argument above in Step I to prove that

$$
h \equiv 0 .
$$

Thus, recalling from (2.19) that $h$ is bounded on $\left(S, g_{S}\right)$, let $\left\{x_{i}\right\}$ be a maximizing sequence for $h$. It follows as before that $\Delta h\left(x_{i}\right) \leq \epsilon_{i},|d h|\left(x_{i}\right) \leq \epsilon_{i}$ while $|d l o g u|\left(x_{i}\right)$ remains uniformly bounded.

To prove $(2.25)$, it is most convienient to pass to the limit of the pointed sequence $\left(S, g_{S}, x_{i}\right)$ by use of Lemmas 1.3-1.4. Thus, a subsequence of $\left\{\left(S, g_{S}, x_{i}\right)\right\}$ converges smoothly, (passing to covers if necessary in the case of collapse), to a complete stationary vacuum solution $\left(S_{\infty}, g_{\infty}, x_{\infty}\right)$. Here the limit potentials $u_{\infty}$ and $\phi_{\infty}$ are limits of the renormalized potentials $u_{i}=u / u\left(x_{i}\right), \phi_{i}=\phi / u\left(x_{i}\right)^{2}$. Observe that $h$ and $d \log u$ are invariant under such renormalizations, as is the right side of (2.24) under the changes $u \rightarrow u_{i}, \bar{g}_{S} \rightarrow \bar{g}_{i}=u_{i}^{2} \cdot g_{S}$.

It follows from these estimates and (2.24), together with the maximum principle, that the limit $\left(S_{\infty}, g_{\infty}, x_{\infty}, u_{\infty}, \phi_{\infty}\right)$ satisfies

$$
h \equiv h_{\infty}=\text { const }, \quad|\bar{\nabla} D E|=0, \quad|\bar{r}|^{2}-\bar{s}^{2}=0,
$$

where $\bar{g}_{\infty}=u_{\infty}^{2} \cdot g_{\infty}$ and

$$
h_{\infty}=\sup _{S} h
$$

To see that $h_{\infty}=0,(2.26)$ and (2.10) imply that $\bar{\nabla} \bar{r}=0$, i.e. the Ricci curvature $\bar{r}_{\infty}$ of $\bar{g}_{\infty}$ is parallel. By the Bianchi identity this implies that the scalar curvature $\bar{s}_{\infty}$ of $\bar{g}_{\infty}$ is constant. Since $h=h_{\infty}$ is constant, (2.18) shows that $u_{\infty}$ is also constant on $\left(S_{\infty}, g_{\infty}\right)$. Hence by (2.4) on $\left(S_{\infty}, g_{\infty}\right)$, it follows that $d \phi_{\infty}=0$. By the definition of $h$ in $(2.18)$, this of course gives $h_{\infty} \equiv 0$, which by (2.27) gives (2.25).

The equation (2.25) means that $u$ is a constant function and $\omega=0$, so that $d \theta=0$. It follows that $\left(S, g_{S}\right)$ and $\left(M, g_{M}\right)$ are both flat, which proves the result.

\section{Proof of Theorem 0.2 .}

The following result gives Theorem 0.2 essentially as an immediate corollary. The proof is a standard consequence of the global result in Theorem 0.1 , together with the control on moduli of stationary vacuum solutions given in Lemmas 1.3 and 1.4.

Theorem 3.1. Let $\left(M, g_{M}\right)$ be a stationary vacuum solution, with orbit data $\left(S, g_{S}, u, \theta\right)$, and $U \subset \subset S$ a domain with smooth boundary, so that $u>0$ on $\bar{U}$. Then there is an (absolute) constant $K<\infty$, independent of $\left(M, g_{M}\right)$ and $U$, such that for all $x \in U$,

$$
\left|r_{S}\right|(x) \leq \frac{K}{\rho(x)^{2}}
$$

where $\rho(x)=\operatorname{dist}_{g_{S}}(x, \partial U)$. 
Proof: The proof is by contradiction. Thus, assume that (3.1) does not hold. Then there are stationary vacuum solutions $\left(M_{i}, g_{M_{i}}\right)$, with orbit data $\left(S_{i}, g_{S_{i}}, u_{i}, \omega_{i}\right)$, smooth domains $U_{i} \subset \subset S_{i}$ on which $u_{i}>0$ and points $x_{i} \in U_{i}$ such that

$$
\rho^{2}\left(x_{i}\right)\left|r_{i}\right|\left(x_{i}\right) \rightarrow \infty, \text { as } i \rightarrow \infty .
$$

Let $\rho_{i}=\rho\left(x_{i}\right)$. Since it may not be possible to choose the points $x_{i}$ so that they maximize $\left|r_{i}\right|$ (over large domains), we shift the base points $x_{i}$ as follows; compare with the proof of Lemma 1.5. Choose $t_{i} \in\left[0, \rho_{i}\right)$ such that

$$
t_{i}^{2} \sup _{B_{x_{i}}\left(\rho_{i}-t_{i}\right)}\left|r_{i}\right|=\sup _{t \in\left[0, \rho_{i}\right)} t^{2} \cdot \sup _{B_{x_{i}}\left(\rho_{i}-t\right)}\left|r_{i}\right| \rightarrow \infty, \text { as } i \rightarrow \infty,
$$

where the last estimate follows from (3.2), (set $\left.t=\rho_{i}\right)$. Let $y_{i} \in B_{x_{i}}\left(\rho_{i}-t_{i}\right)$ be points such that

$$
\left|r_{i}\right|\left(y_{i}\right)=\sup _{B_{x_{i}}\left(\rho_{i}-t_{i}\right)}\left|r_{i}\right| \text {. }
$$

Further, setting $t=t_{i}\left(1-\frac{1}{k}\right), k>1$, in (3.3), one obtains the estimate

$$
t_{i}^{2}\left|r_{i}\right|\left(y_{i}\right) \geq t_{i}^{2}\left(1-\frac{1}{k}\right)^{2} \cdot \sup _{B_{x_{i}}\left(\rho_{i}-t_{i}\left(1-\frac{1}{k}\right)\right)}\left|r_{i}\right| \geq t_{i}^{2}\left(1-\frac{1}{k}\right)^{2} \cdot \sup _{B_{y_{i}}\left(t_{i} / k\right)}\left|r_{i}\right|,
$$

so that

$$
\sup _{B_{y_{i}}\left(t_{i} / k\right)}\left|r_{i}\right| \leq\left(1-\frac{1}{k}\right)^{-2}\left|r_{i}\right|\left(y_{i}\right),
$$

Now rescale or blow-up the metric so that $\left|\widetilde{r}_{i}\right|\left(y_{i}\right)=1$ by setting $\widetilde{g}_{i}=\left|r_{i}\right|\left(y_{i}\right) \cdot g$, and consider the pointed sequence $\left(U_{i}, \widetilde{g}_{i}, y_{i}\right)$. We have

$$
\left|\widetilde{r}_{i}\right|\left(y_{i}\right)=1
$$

and by (3.3) and scale invariance,

$$
\operatorname{dist}_{\widetilde{g}_{i}}\left(y_{i}, \partial U_{i}\right) \rightarrow \infty, \text { as } i \rightarrow \infty \text {. }
$$

Also, (compare with (1.21)), it follows from (3.6) that

$$
\left|\widetilde{r}_{i}\right|(x) \leq C\left(\operatorname{dist}_{\widetilde{g}_{i}}\left(x, y_{i}\right)\right)
$$

We also normalize $u$ by setting

$$
\tilde{u}_{i}(x)=\frac{u(x)}{u\left(y_{i}\right)}
$$

and note that $\tilde{u}_{i}>0$ on $U_{i}$.

We may now apply Lemmas 1.3 and 1.4, exactly as in the proof of Lemma 1.5 to conclude that a subsequence of the pointed sequence $\left(U_{i}, \tilde{g}_{i}, \tilde{u}_{i}, \tilde{\omega}_{i}, y_{i}\right)$ converges in the $C^{\infty}$ topology on compact subsets, to a limit stationary vacuum solution $\left(U_{\infty}, \widetilde{g}_{\infty}, \tilde{u}_{\infty}, \tilde{\omega}_{\infty}, y\right)$, which is complete and satisfies $\tilde{u}_{\infty}>0$ everywhere. Here, one must pass to the universal cover in case of collapse, as in Lemma 1.4, and the potential $\tilde{u}_{i}$ and 1-form $\tilde{\omega}_{i}$ are normalized so that $\tilde{u}_{i}\left(y_{i}\right)=1$ and $\left|\tilde{\omega}_{i}\left(y_{i}\right)\right|$ is bounded.

By Theorem 0.1, $\widetilde{g}_{\infty}$ must be flat, $\tilde{u}_{\infty}$ constant and $d \tilde{\omega}_{\infty}=0$. However, the smooth convergence of the sequence $\left(U_{i}, \widetilde{g}_{i}\right)$ guarantees that the equality (3.7) passes to the limit, contradicting the fact that $\widetilde{g}_{\infty}$ is flat.

As in the proof of Lemmas 1.3 and 1.4, it follows from (3.1) that

$$
|d \log u|(x) \leq \frac{K}{\rho(x)}
$$

and

$$
u^{-2}|\omega|(x) \leq \frac{K}{\rho(x)}
$$


Combining the estimates (3.1) and (3.11)-(3.12), one obtains the same bound on the full curvature tensor $R_{M}$ of $(M, g)$.

Note that since $K$ is independent of the domain $U$, (3.1) holds for $\rho$ the distance to the boundary $\Sigma$ of $S$, even if $\Sigma$ is singular. To see this, just apply Theorem 3.1 to a smooth exhaustion $U_{j}$ of $S$, with $\partial U_{j}$ converging to $\partial S$ in the Hausdorff metric on subsets of $\left(S, g_{S}\right)$. In particular, these results together prove Theorem 0.2 .

We note that elliptic regularity further implies that, for any $j \geq 1$,

$$
\left|\nabla^{j} R_{M}\right|(x) \leq \frac{K(j)}{\rho^{2+j}(x)},\left|\nabla^{j} \log u\right|(x) \leq \frac{K(j)}{t^{j}(x)} .
$$

Theorem 0.2 , when combined with Lemmas 1.3 and 1.4, shows that the moduli space of stationary vacuum solutions is apriori well-controlled away from the boundary $\Sigma=\partial S$. Thus, away from the boundary, sequences of such metrics either have a smoothly convergent subsequence, or they collapse, in which case the universal covers have a convergent subsequence.

Theorems 0.1 and 0.2 give new proofs of similar results for static vacuum solutions in [An2, Thm. 3.2]. Similarly, in work to follow, we plan to generalize the results on the asymptotic structure of static vacuum space-times in [A1] to stationary space-times as well as consider the Riemannian analogues of these questions.

\section{REFERENCES}

[A1] M. Anderson, On the structure of solutions to the static vacuum Einstein equations, (preprint, S.U.N.Y. Stony Brook, July 1998), http://www.math.sunysb.edu/ anderson

[A2] M. Anderson, Scalar curvature, metric degenerations and the static vacuum Einstein equations on 3manifolds I, Geom. and Funct. Anal., vol 9:5, (1999), 855-967; http://www.math.sunysb.edu/ anderson

[A3] M. Anderson, Extrema of curvature functionals on the space of metrics on 3-manifolds, Calc. Var. and P.D.E., vol. 5, (1997), 199-269.

[B] A. Besse, Einstein Manifolds, Springer Verlag, New York, (1987).

[C] J. Cheeger, Finiteness theorems for Riemannian manifolds, Amer. Jour. Math., vol. 92, (1970), 61-75.

[CG1] J. Cheeger and M. Gromov, Collapsing Riemannian manifold while keeping their curvature bounded I, Jour. Diff. Geom., vol. 23, (1986), 309-346.

[CG2] J. Cheeger and M. Gromov, Collapsing Riemannian manifold while keeping their curvature bounded II, Jour. Diff. Geom., vol. 32, (1990), 269-298.

[EL] J. Eells and L. Lemaire, A report on harmonic maps, Bull. London Math. Soc., vol. 10, (1978), 1-68.

[F] K. Fukaya, Hausdorff convergence of Riemannian manifolds and its applications, Recent. Topics in Diff. and Analytic Geom. (T. Ochiai, ed.), Kinokuniya, Tokyo, (1990), 143-283.

[GT] D. Gilbarg and N. Trudinger, Elliptic Partial Differential Equations of $2^{\text {nd }}$ Order, $2^{\text {nd }}$ Edition, Springer Verlag, New York, (1983).

[Ha] S. Harris, Conformally stationary space-times, Class. Quantum Gravity, vol. 9, (1992), 1823-1827.

[H1] M. Heusler, Black Hole Uniqueness Theorems, Cambridge U. Press, Cambridge, (1996).

[H2] M. Heusler, Stationary Black Holes: Uniqueness and Beyond, Living Reviews in Relativity, vol. 1, No. 6, (1998): http://www.livingreviews.org

[Ka] A. Kasue, A convergence theorem for Riemannian manifolds and some applications, Nagoya Math. J., vol. $114,(1989), 21-51$.

[Kr] D. Kramers, H.Stephani, M. MacCallum, E. Herlt, Exact Solutions of Einstein's Field Equations, Cambridge U. Press, Cambridge, (1980).

[L] A. Lichnerowicz, Theories Relativistes de la Gravitation et de L'Electromagnetisme, Masson and Cie., Paris, (1955).

[MTW] C.W.Misner, K.S. Thorne and J.A. Wheeler, Gravitation, W.H. Freeman, New York, (1973).

[O] P. Orlik, Seifert Manifolds, Lecture Notes in Math., vol. 291, Springer Verlag, New York, (1972).

[P] P. Petersen, Riemannian Geometry, Grad. Texts in Math., vol. 171, Springer Verlag, New York (1998).

[Ri] W. Rindler, Essential Relativity, $2^{\text {nd }}$ Edition, Springer Verlag, New York, (1977).

[Ro] X. Rong, The limiting eta invariants of collapsed three-manifolds, Jour. Diff. Geom., vol. 37, (1993), 535-568. 
[T] M. Taylor, Partial Differential Equations III, Applied Math. Sciences, vol. 117, Springer Verlag, New York, 1996.

[W] R. Wald, General Relativity, Univ. of Chicago Press, Chicago (1984).

October 1999/March 2000

Department of Mathematics

S.U.N.Y. at Stony Brook

Stony Brook, N.Y. 11794-3651

anderson@math.sunysb.edu 